\title{
Beyond Bacteria:
}

\section{Bacteriophage-Eukaryotic Host Interactions Reveal Emerging Paradigms of Health and Disease}

\author{
Anushila Chatterjee and Breck A. Duerkop* \\ Department of Immunology and Microbiology, University of Colorado School of Medicine, Aurora, CO, United States
}

For decades, a wealth of information has been acquired to define how host associated microbial communities contribute to health and disease. Within the human microbiota this has largely focused on bacteria, yet there is a myriad of viruses that occupy various tissue sites, the most abundant being bacteriophages that infect bacteria. Animal hosts are colonized with niche specific microbial communities where bacteria are continuously co-evolving with phages. Bacterial growth, metabolic activity, pathogenicity, antibiotic resistance, interspecies competition and evolution can all be influenced by phage

OPEN ACCESS

Edited by: Irah L. King,

McGill University, Canada

Reviewed by:

Jeremy J. Barr,

Monash University, Australia

Kimberley Seed,

University of California, Berkeley,

United States

*Correspondence:

Breck A. Duerkop

breck.duerkop@ucdenver.edu

Specialty section: This article was submitted to Microbial Immunology, a section of the journal Frontiers in Microbiology

Received: 01 April 2018 Accepted: 07 June 2018

Published: 27 June 2018

Citation:

Chatterjee A and Duerkop BA (2018)

Beyond Bacteria:

Bacteriophage-Eukaryotic Host Interactions Reveal Emerging Paradigms of Health and Disease.

Front. Microbiol. 9:1394. doi: 10.3389/fmicb.2018.01394 infection and the beneficial nature of such interactions suggests that to an extent phages are tolerated by their hosts. With the understanding that phage-specific hostmicrobe interactions likely contribute to bacterial interactions with their mammalian hosts, phages and their communities may also impact aspects of mammalian health and disease that have gone unrecognized. Here, we review recent progress in understanding how bacteria acquire and tolerate phage in both pure culture and within complex communities. We apply these findings to discuss how intra-body phages interact with bacteria to influence their eukaryotic hosts through potential contributions to microbial homeostasis, mucosal immunity, immune tolerance and autoimmunity.

Keywords: bacteriophage, virome, host-microbe interactions, phage-bacteria interactions, microbiota, microbiome, phage immunity

\section{INTRODUCTION}

The human body hosts a complex and dynamic consortia of microbes consisting of bacteria, archaea, fungi, viruses and protozoa (Zou et al., 2016; Blum, 2017; Chabe et al., 2017; Huseyin et al., 2017; Koskinen et al., 2017; Raymann et al., 2017). Among the core members of the human microbiota, bacteria have garnered significant attention because of their contributions to human physiology and disease (Hooper and Gordon, 2001; Belkaid and Hand, 2014; Byrd et al., 2018; Zhao and Elson, 2018). The emergence of culture-independent approaches and techniques for viral enrichments from complex microbial samples has identified a vast consortium of understudied viruses within host associated microbiotas (Virgin, 2014).

The onset of the "omics" revolution led by $16 \mathrm{~s}$ rDNA sequencing rapidly advanced our ability to survey the bacterial component of the microbiota in unprecedented detail. Extending from these studies, the implementation of metagenomic DNA sequencing revealed a robust viral component to the microbiota and identified bacteriophages (phages) as dominant members. In humans, 
phages populate most surfaces including skin (Foulongne et al., 2012; Oh et al., 2016), the oral cavity (Willner et al., 2011; Pride et al., 2012; Abeles et al., 2014), lungs (Willner et al., 2009; Dickson and Huffnagle, 2015), the intestine (Reyes et al., 2010; Minot et al., 2011; Manrique et al., 2016) and the urinary tract (Santiago-Rodriguez et al., 2015; MillerEnsminger et al., 2018). Phage-bacteria interactions have been studied in varying detail in vitro (Chevallereau et al., 2016; Leskinen et al., 2016; Mojardin and Salas, 2016), however, little work to date has revealed insights into how phages interact with their bacterial hosts in human and animal systems. Body sites are endowed with unique characteristics including microenvironments that can define unique physiologies, thus it is conceivable that in some instances phage-bacteria interactions in vivo may be distinct from what has been studied in the laboratory.

Within the human body, phages infect bacterial hosts and undergo lytic replication and phage particle biogenesis to synthesize new infectious phages or integrate into the host bacterial genome as quiescent lysogenic prophages that are propagated vertically during cell division (Weinbauer, 2004; Hobbs and Abedon, 2016). Environmental cues such as nutrients, antibiotics and reactive oxygen species are well documented in vitro inducers of prophage excision from bacterial genomes, yet we know very little about the in vivo cues that promote prophage excision or those that influence the maintenance of lysogeny (DeMarini and Lawrence, 1992; Duerkop et al., 2012; Matos et al., 2013).

Considering the plethora of lytic and lysogenic phages that associate with humans, these phages are poised to have a significant impact on human physiology during both health and disease. In fact, research using animal models indicate that the intestinal microbiota promotes phage genome evolution allowing phages to infect naïve bacterial species and consequently fostering intra-body persistence (De Sordi et al., 2017). Hence, the cross-talk between resident bacteria and phages potentially contributes to the maintenance of microbial homeostasis within the human body. In this review, we will discuss phagebacterial interactions within the context of host-associated microbial communities and will explore the underlying reasons for the evolution of phage tolerance in both bacteria and animals.

\section{PHAGE-BACTERIAL COLLABORATION: BENEFITS OF BEFRIENDING THE ENEMY}

Predatory lytic phages play crucial roles in maintaining diversity within microbial ecosystems (Maslov and Sneppen, 2017). Lytic phages adsorb to susceptible bacteria and subsequently infect and kill these bacteria. According to the classic "kill-thewinner" model, abundant bacterial species in a population have a greater possibility of encountering virulent phages and consequently face death, thus preventing niche monopoly by a single bacterial species (Thingstad, 2000; Rodriguez-Brito et al., 2010). Coculture studies using two competitive Pseudomonas strains demonstrated that phages enable the less competitive bacterial species to persist by infecting the more dominant species at a higher frequency, thus influencing community composition (Brockhurst et al., 2006). The contribution of lytic phages to bacterial diversity and richness in host associated environments is unknown and it is unclear whether bonafide "kill-the-winner" dynamics apply (Reyes et al., 2010; Allen et al., 2011; Abedon, 2012).

Lysogenic phages integrated into host bacterial chromosomes can constitute up to $20 \%$ of bacterial genomes (Casjens, 2003), raising the question of why some bacteria tolerate such high burdens of phage DNA? Phage tolerance is likely supported by the potential positive outcomes bestowed upon the bacterium while harboring the viral DNA (Figure 1). Specifically, bacteria have co-evolved with their phages to benefit from the inclusion of viral genes within their genomes, which can aid in bacterial fitness, pathogenesis and adaptation to changing environments. Within this context, we will briefly discuss how bacteria benefit from their associated phages and we direct the readers to more recent comprehensive reviews on this subject (Roossinck, 2011; Obeng et al., 2016; Harrison and Brockhurst, 2017; Touchon et al., 2017).

Phages are vehicles for the horizontal transfer of genes which upon acquisition can influence individual and bacterial community phenotypes (reviewed in Touchon et al., 2017). For instance, prophages have been shown to confer pathogenic and antibiotic resistance traits for their bacterial hosts (Matos et al., 2013; Obeng et al., 2016; Lekunberri et al., 2017). Examples include a myriad of toxins which are encoded within prophage elements, the most well studied being the Shiga toxin-encoding prophages of Escherichia coli (STEC) which cause fatal gastrointestinal infections in humans (Shaikh and Tarr, 2003). Interestingly, a recent report showed that in addition to toxin production, the carriage of Shiga toxinencoding prophages enhances antimicrobial tolerance of STEC by modifying the bacterium's metabolism (Holt et al., 2017). Additionally, toxin encoding phages from numerous pathogenic bacteria have been demonstrated to transduce and lysogenize non-pathogenic bacteria, converting them to virulent strains (Faruque et al., 1999; Schmidt et al., 1999; Broudy and Fischetti, 2003).

The induction of lysogenic prophages from bacterial chromosomes has been linked to pathogen fitness and virulence. Production of EfCIV583, a satellite prophage who's DNA is packaged into the capsid of the helper phage vB_EfaS_V583-P1 in Enterococcus faecalis strain V583, allows the host bacterium to compete with non-lysogenic peers (Duerkop et al., 2012; Matos et al., 2013). Another example from mixed culture experiments shows that prophage excision and subsequent phage mediated lysis of a subpopulation of host bacteria results in the timed release of bacteriocins that kill bacterial competitors and clear the niche for the phage-harboring bacteria (Nedialkova et al., 2016). Spontaneous prophage induction can also prime a shift in bacterial lifestyle from independently growing bacterial cells to organized cellular aggregates termed biofilms (reviewed in Nanda et al., 2015). The lungs of cystic fibrosis (CF) patients are colonized by sessile communities of Pseudomonas aeruginosa whose structural organization resemble 


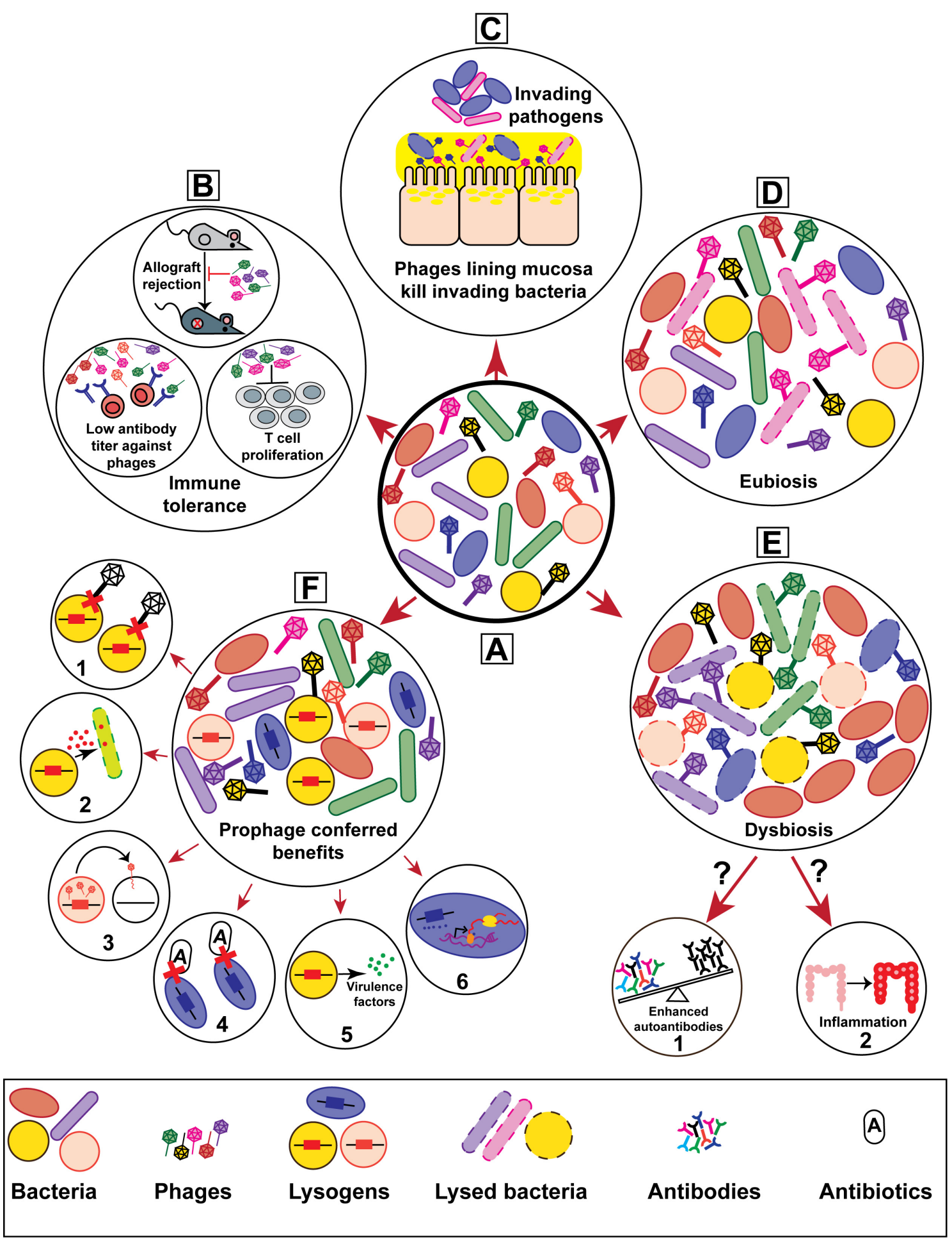

FIGURE 1 | Bacteriophages contribute to the genetic and physiological traits of their hosts thereby influencing host-microbe interactions. (A) Schematic representation of a healthy mammalian microbiota consisting of heterogeneous phage and bacterial populations. The prolonged and ubiquitous presence of phages in mammalian microbiotas are hypothesized to have considerable effects on health and disease. Phage-driven impacts on mammalian hosts include (B) immune tolerance, (C) mucosal immunity, and (D) homeostatic eubiosis. Altered phage diversity and richness have been suggested to drive (E) bacterial dysbiosis, potentially leading to (E-1) autoimmune progression in type I diabetes and (E-2) inflammation during inflammatory bowel disease. On the other hand, phage infection endows bacteria with multiple features that alter bacterial interactions with their mammalian hosts. (F) Lysogenic conversion via the acquisition of prophages can increase bacterial host fitness. Prophage provided traits include (F-1) superinfection immunity, (F-2) elimination of bacterial competitors, (F-3) horizontal gene transfer, (F-4) enhanced antibiotic resistance, (F-5) virulence, and (F-6) altered gene expression. 
biofilms (Bjarnsholt et al., 2013). Within these P. aeruginosa biofilms prophage induction occurs resulting in the release of the filamentous phage Pf4 (Secor et al., 2017). Pf4 phages promote biofilm assembly, facilitate persistence of the host bacteria in the lung and modulate inflammatory responses to promote chronic infections (Rice et al., 2009; Secor et al., 2015, 2017). Hence, prophage induction and subsequent phage-driven bacterial cell lysis provides a fitness benefit to the host bacterial community.

The integration of lysogenic phages into bacterial chromosomes can disrupt genes, thus altering phenotypes and in some cases altering bacterial fitness (Coleman et al., 1991; Bernhardt et al., 2000; Brussow et al., 2004). In a phenomenon termed reversible active lysogeny, prophage excision from the bacterial genome re-activates a host gene without activating the phages lytic cycle which promotes host adaptation (Feiner et al., 2015). For example, a Listeria monocytogenes prophage integrated within the master regulator of competence gene $\operatorname{comK}$, during intracellular growth excises from the L. monocytogenes genome to restore the $\operatorname{com} K$ reading frame. The bacterium represses phage lysis and produces a functional ComK protein to promote immune evasion (Rabinovich et al., 2012). In the case of reversible active lysogeny, prophages provide gainof-function phenotypes at the cost of the bacterium which must maintain the prophage element both within its dormant integrated form and repress its lytic functions after excision. Such costs are likely mitigated considering maintenance of these phages benefit the bacterium. The mechanisms driving these types of co-evolution are unclear, however, the acquisition and selection of phages within bacterial genomes shows that tolerance of phage lysogeny can promote context dependent attributes that promote bacterial adaptation to diverse environments.

Bacteria have evolved multiple defense strategies to restrict virulent phage predation and prophage acquisition (Labrie et al., 2010). Examples include restriction-modification (RM) and CRISPR (clustered regularly interspaced short palindromic repeats)/Cas (CRISPR-associated protein) systems that target invading phage DNA for destruction (Tock and Dryden, 2005; Barrangou et al., 2007; Sorek et al., 2008; Abedon, 2012). The absence of functional CRISPR-Cas has been linked to the emergence of multidrug resistant bacteria (Palmer and Gilmore, 2010; van Belkum et al., 2015), suggesting that the inability of bacteria to protect their genomes from foreign mobile elements including phages promotes bacterial adaptation to defined environmental conditions. Conversely, a recent study demonstrated that a conditional CRISPR/Cas system of Staphylococcus epidermidis evolved to favor prophage acquisition promoting a type of selective phage tolerance by degrading lytic phage DNA but allowing phage lysogeny (Goldberg et al., 2014) and RM systems have been shown to advocate prophage acquisition by postponing the onset of viral replication until bacterial population density has reached a point where the probability of lysogenic conversion is high (Pleska et al., 2018). These studies suggest that even in the presence of functional CRISPR-Cas and RM systems there may be preferences for specific DNAs that benefit bacteria in a context dependent manner.

\section{BACTERIOPHAGE-EUKARYOTE INTERACTIONS: THE TIP OF THE ICEBERG}

Although metagenomic studies have revealed phages as one of the most abundant components of the human microbiota (Reyes et al., 2010; Minot et al., 2011; Foulongne et al., 2012; Pride et al., 2012; Oh et al., 2014; Dickson and Huffnagle, 2015; Santiago-Rodriguez et al., 2015; Oh et al., 2016), information on the interactions of phages with animal cells and how phages contribute to health and disease is limited. In this section, we will discuss recent literature related to how phages interact with eukaryotic hosts and how these interactions influence host immunity.

Over a decade ago, it was appreciated that intestinal phages breach the physical barrier of the mammalian intestine (Górski et al., 2006b). Compromised intestinal epithelial integrity during inflammation provides phages with access to the bloodstream and consequently their spread to different tissues (Handley et al., 2012). However, in the absence of intestinal barrier distress phages still migrate to host restricted sites such as peripheral blood and organs. It has been proposed that phages are naturally internalized into eukaryotic cells (Duerkop and Hooper, 2013; Tian et al., 2015; Zhang et al., 2017), however, the mechanisms behind phage uptake by eukaryotic cells are just beginning to be explored. A long held theory behind phage internalization by eukaryotic cells is that these events were preceded by the entry of phage-infected bacterial cells that transport phages (Hsia et al., 2000; Johnston, 2002; Duerkop and Hooper, 2013). Recently phages have been shown to gain access to epithelial cells directly through active transcytosis in the absence of their bacterial host (Nguyen et al., 2017). Phages were shown to permeate the apical surface of epithelial cells via endocytosis, become compartmentalized and finally exocytosed through the basal side of the cell (Nguyen et al., 2017). In a separate study, Escherichia coli phage PK1A2 was shown to recognize and bind neuroblastoma cells displaying polysialic acids on their cell surface (Lehti et al., 2017). Following adhesion, phage PK1A2 is internalized by the endolysosomal pathway and are eventually degraded in the lysosome (Lehti et al., 2017). It is hypothesized that during these internalization events, phages may escape lysosomal destruction and potentially create opportunities for trans-kingdom genetic exchange or stimulate cellular immunity (Duerkop and Hooper, 2013; Lehti et al., 2017). In another example of potential trans-kingdom interactions, it has been proposed that phages act as an additional layer of nonhost derived immunity against incoming pathogens at mucosal surfaces by binding to mucin glycoproteins [(Barr et al., 2013a) and discussed later]. Carbohydrate modifications, including sialic acids and various glycosylations are abundant in host derived mucins (Royle et al., 2008). Determining if phage adhesion to the sugar epitopes of host mucins is a common strategy by which phages interact with eukaryotic mucosal surfaces should 
reveal possible mechanisms behind the in vivo translocation and dispersal of phages within the human body.

As phages are significant reservoirs of genetic diversity and considering phages are capable of entering eukaryotic cells, this raises questions about the possibility of bidirectional transkingdom gene exchange between phages and their animal hosts. Multicellular eukaryotes have been reported to harbor phage capsid gene orthologs in their genomes that resemble phages of the obligate intracellular pathogen Chlamydophila pneumoniae (Rosenwald et al., 2014). Phages have also been implicated in the dissemination of bacterial aerolysin and lysozyme genes within eukaryotic hosts (Moran et al., 2012; Metcalf et al., 2014). Conversely, metazoan-like gene modules whose functions have yet to be defined have been found in phages of the insect parasite Wolbachia (Bordenstein and Bordenstein, 2016). Together, these observations suggest the potential for the genetic interplay between phages and eukaryotes that contribute to trans-kingdom evolution (Figure 1).

Commensal bacteria regulate various facets of host immunity, yet there are significant gaps in our understanding of the mechanisms driving microbiota mediated immune regulation (Mazmanian et al., 2005; Ivanov et al., 2008; Arpaia et al., 2013; Fung et al., 2017; Novince et al., 2017; Schnupf et al., 2017). Considering phages influence the assembly of microbial communities and modulate bacterial diversity in various ecosystems (Barr et al., 2013b; Koskella and Meaden, 2013), perhaps phage-bacteria interactions can direct the host immune response. Phages could potentially modulate immune interactions between a eukaryotic host and its microbiota by providing novel traits within subpopulations of bacteria or by causing shifts in the resident bacterial community composition through targeted killing of defined community members (Figure 1). Given the prevalence of phages at multiple tissue sites within the human body, it is likely that phages play an unrecognized role in promoting the development and activity of the immune system through interactions with their host bacteria. It is possible that lytic phages could directly stimulate antiviral innate immunity by engaging nucleic acid sensors or inadvertently by killing their bacterial hosts and releasing soluble bacterial antigens that stimulate pattern recognition receptors. If either of these scenarios were true, this would have profound implications for the development of lytic phages as antibacterial therapeutics.

Changes in phage community composition occur during human disease. For example, the diversity and composition of intestinal phages is significantly different between healthy individuals and patients with inflammatory bowel diseases (IBD) (Wagner et al., 2013; Norman et al., 2015). Individuals with IBD have reduced enteric bacterial diversity relative to the healthy individuals. However, alterations in bacterial richness does not always correlate with the dramatic phage expansion associated with IBD (Norman et al., 2015), suggesting signals from the immune system may directly influence phage abundances. Additionally, alterations in enteric phage populations was observed prior to the development of autoantibodies in children who were predisposed to develop Type I diabetes (Zhao et al., 2017). These disease-related shifts in phage community composition suggest a potential role for intestinal phages in the development of bacterial dysbiosis. Although phages have been implicated in diseases associated with bacterial dysbiosis, it is unclear if phages directly contribute to inflammation and autoimmune disease by altering microbial homeostasis (Figure 1).

The collaboration between phages and their animal hosts to eliminate deleterious bacteria is opening new avenues for the study of host-microbe interactions during health and disease. In a recent study, researchers revealed a neutrophil-phage alliance that together cleared multi-drug resistant $P$. aeruginosa in a lung infection mouse model (Roach et al., 2017). This study suggests that host innate immunity may be more effective at clearing pathogenic bacteria with help from lytic phages. In vitro tissue culture studies suggest that phages protect epithelial mucosal surfaces from invading bacteria (Barr et al., 2013a, 2015). These studies propose that phages with binding affinity for host mucins form a protective antibacterial defense called BAM (bacteriophage adhesion to mucus) which serves as a nonhost derived innate immunity at mucosal surfaces. According to the BAM model, adhesion of phages to mucin glycoproteins and subsequent subdiffusive movement through the mucus layer concentrates phages at mucosal surfaces. An enrichment of phages in the mucosa may provide protection against bacterial invaders and limit pathogen colonization (Barr et al., 2013a, 2015). Although, these findings suggest that phages contribute to host defenses (Figure 1), their function in promoting mucosal health remain to be explored.

An increasing body of data suggests that phages engage in interactions with mammalian immune cells and modulate different aspects of host immune responses. Phages are weakly immunogenic and the adaptive immune system produces low titers of phage-neutralizing antibodies without mounting an inflammatory response (Dabrowska et al., 2006; Górski et al., 2012; An et al., 2014; Majewska et al., 2015). Knowing that phages are ubiquitous within host associated microbiotas and possibly within the host systemic environment, it is possible that immune tolerance to phages occurs due to the continued exposure of the immune system to phages. Several studies propose a role for phages in promoting immune tolerance by downregulating $\mathrm{T}$ cell proliferation, through the reduction of antibody production and in the prevention of allogenic transplant rejection in animal models (Górski et al., 2006a,b, 2007; McVay et al., 2007). For a comprehensive discussion on the effect of phages on immunemodulation readers are directed to a recent review by Górski et al. (2017).

\section{CONCLUDING REMARKS}

Phages endow their host bacteria with competitive traits, facilitate adaptation for the colonization of new niches and promote bacterial evolution. It is becoming increasingly clear that the impact of phages extend beyond their bacterial hosts and their potential influences on human health are just beginning to be explored (De Paepe et al., 2014; Norman et al., 2015; Manrique et al., 2016; Wahida et al., 2016). Recent studies 
bring to light concepts for how bacteria and animals have coevolved to tolerate phages through beneficial interactions that may dictate the outcomes of host-microbe associations. Through these interactions phages and their communities hold substantial promise as modulators of human health and disease.

Moving forward, studies that employ modern "omics" technologies to study the microbiota such as metagenomics, transcriptomics and proteomics should by default incorporate analyses of phage communities. Alongside such studies, researchers must make efforts through the use of mouse models and in vivo defined microbial communities to move beyond descriptive studies and begin providing mechanistic details into how phages interact with host-associated bacteria and how these interactions influence immunity and physiology. Furthermore, specific attention should be given to the mammalian host responses that drive the assembly of phage community composition within the microbiota and how

\section{REFERENCES}

Abedon, S. T. (2012). Bacterial 'immunity' against bacteriophages. Bacteriophage 2, 50-54. doi: 10.4161/bact.18609

Abeles, S. R., Robles-Sikisaka, R., Ly, M., Lum, A. G., Salzman, J., Boehm, T. K., et al. (2014). Human oral viruses are personal, persistent and gender-consistent. ISME J. 8, 1753-1767. doi: 10.1038/ismej.2014.31

Allen, H. K., Looft, T., Bayles, D. O., Humphrey, S., Levine, U. Y., Alt, D., et al. (2011). Antibiotics in feed induce prophages in swine fecal microbiomes. mBio 2:e00260-11. doi: 10.1128/mBio.00260-11

An, T. W., Kim, S. J., Lee, Y. D., Park, J. H., and Chang, H. I. (2014). The immune-enhancing effect of the Cronobacter sakazakii ES2 phage results in the activation of nuclear factor-kappaB and dendritic cell maturation via the activation of IL-12p40 in the mouse bone marrow. Immunol. Lett. 157, 1-8. doi: 10.1016/j.imlet.2013.10.007

Arpaia, N., Campbell, C., Fan, X., Dikiy, S., van der Veeken, J., deRoos, P., et al. (2013). Metabolites produced by commensal bacteria promote peripheral regulatory T-cell generation. Nature 504, 451-455. doi: 10.1038/nature12726

Barr, J. J., Auro, R., Furlan, M., Whiteson, K. L., Erb, M. L., Pogliano, J., et al. (2013a). Bacteriophage adhering to mucus provide a non-host-derived immunity. Proc. Natl. Acad. Sci. U.S.A. 110, 10771-10776. doi: 10.1073/pnas. 1305923110

Barr, J. J., Youle, M., and Rohwer, F. (2013b). Innate and acquired bacteriophagemediated immunity. Bacteriophage 3:e25857. doi: 10.4161/bact.25857

Barr, J. J., Auro, R., Sam-Soon, N., Kassegne, S., Peters, G., Bonilla, N., et al. (2015). Subdiffusive motion of bacteriophage in mucosal surfaces increases the frequency of bacterial encounters. Proc. Natl. Acad. Sci. U.S.A. 112, 1367513680. doi: $10.1073 /$ pnas. 1508355112

Barrangou, R., Fremaux, C., Deveau, H., Richards, M., Boyaval, P., Moineau, S., et al. (2007). CRISPR provides acquired resistance against viruses in prokaryotes. Science 315, 1709-1712. doi: 10.1126/science. 1138140

Belkaid, Y., and Hand, T. W. (2014). Role of the microbiota in immunity and inflammation. Cell 157, 121-141. doi: 10.1016/j.cell.2014. 03.011

Bernhardt, T. G., Roof, W. D., and Young, R. (2000). Genetic evidence that the bacteriophage phi X174 lysis protein inhibits cell wall synthesis. Proc. Natl. Acad. Sci. U.S.A. 97, 4297-4302. doi: 10.1073/pnas.97.8.4297

Bjarnsholt, T., Alhede, M., Alhede, M., Eickhardt-Sorensen, S. R., Moser, C., Kuhl, M., et al. (2013). The in vivo biofilm. Trends Microbiol. 21, 466-474. doi: $10.1016 /$ j.tim.2013.06.002

Blum, H. E. (2017). The human microbiome. Adv. Med. Sci. 62, 414-420. doi: 10.1016/j.advms.2017.04.005

Bordenstein, S. R., and Bordenstein, S. R. (2016). Eukaryotic association module in phage WO genomes from Wolbachia. Nat. Commun. 7:13155. doi: 10.1038/ ncomms 13155 these signals influence phage interactions with their bacterial hosts. Only after these basic questions are explored can we begin to understand how to harness phages for the manipulation of bacterial communities that promote human health.

\section{AUTHOR CONTRIBUTIONS}

All authors listed have made a substantial, direct and intellectual contribution to the work, and approved it for publication.

\section{FUNDING}

This work was supported in part by NIH grant K01DK102436 (BD).

Brockhurst, M. A., Fenton, A., Roulston, B., and Rainey, P. B. (2006). The impact of phages on interspecific competition in experimental populations of bacteria. BMC Ecol. 6:19. doi: 10.1186/1472-6785-6-19

Broudy, T. B., and Fischetti, V. A. (2003). In vivo lysogenic conversion of Tox Streptococcus pyogenes to Tox ${ }^{+}$with Lysogenic Streptococci or free phage. Infect Immun. 71, 3782-3786. doi: 10.1128/IAI.71.7.3782-3786.2003

Brussow, H., Canchaya, C., and Hardt, W. D. (2004). Phages and the evolution of bacterial pathogens: from genomic rearrangements to lysogenic conversion. Microbiol. Mol. Biol. Rev. 68, 560-602. doi: 10.1128/MMBR.68.3.560-602.2004

Byrd, A. L., Belkaid, Y., and Segre, J. A. (2018). The human skin microbiome. Nat. Rev. Microbiol. 16, 143-155. doi: 10.1038/nrmicro.2017.157

Casjens, S. (2003). Prophages and bacterial genomics: what have we learned so far? Mol. Microbiol. 49, 277-300. doi: 10.1046/j.1365-2958.2003.03580.x

Chabe, M., Lokmer, A., and Segurel, L. (2017). Gut protozoa: friends or foes of the human gut microbiota? Trends Parasitol. 33, 925-934. doi: 10.1016/j.pt.2017.08. 005

Chevallereau, A., Blasdel, B. G., De Smet, J., Monot, M., Zimmermann, M., Kogadeeva, M., et al. (2016). Next-Generation “-omics” approaches reveal a massive alteration of host RNA metabolism during bacteriophage infection of Pseudomonas aeruginosa. PLoS Genet. 12:e1006134. doi: 10.1371/journal.pgen. 1006134

Coleman, D., Knights, J., Russell, R., Shanley, D., Birkbeck, T. H., Dougan, G., et al. (1991). Insertional inactivation of the Staphylococcus aureus beta-toxin by bacteriophage phi 13 occurs by site- and orientation-specific integration of the phi 13 genome. Mol. Microbiol. 5, 933-939. doi: 10.1111/j.1365-2958.1991. tb00768.x

Dabrowska, K., Switala-Jelen, K., Opolski, A., and Gorski, A. (2006). Possible association between phages, Hoc protein, and the immune system. Arch. Virol. 151, 209-215. doi: 10.1007/s00705-005-0641-7

De Paepe, M., Leclerc, M., Tinsley, C. R., and Petit, M. A. (2014). Bacteriophages: an underestimated role in human and animal health? Front. Cell. Infect. Microbiol. 4:39. doi: $10.3389 /$ fcimb.2014.00039

De Sordi, L., Khanna, V., and Debarbieux, L. (2017). The gut microbiota facilitates drifts in the genetic diversity and infectivity of bacterial viruses. Cell Host Microbe 22, 801.e3-808.e3. doi: 10.1016/j.chom.2017. 10.010

DeMarini, D. M., and Lawrence, B. K. (1992). Prophage induction by DNA topoisomerase II poisons and reactive-oxygen species: role of DNA breaks. Mutat. Res. 267, 1-17. doi: 10.1016/0027-5107(92)90106-C

Dickson, R. P., and Huffnagle, G. B. (2015). The lung microbiome: new principles for respiratory bacteriology in health and disease. PLoS Pathog. 11:e1004923. doi: 10.1371/journal.ppat.1004923

Duerkop, B. A., Clements, C. V., Rollins, D., Rodrigues, J. L., and Hooper, L. V. (2012). A composite bacteriophage alters colonization by an intestinal commensal bacterium. Proc. Natl. Acad. Sci. U.S.A. 109, 17621-17626. doi: 10.1073/pnas.1206136109 
Duerkop, B. A., and Hooper, L. V. (2013). Resident viruses and their interactions with the immune system. Nat. Immunol. 14, 654-659. doi: 10.1038/ni.2614

Faruque, S. M., Rahman, M. M., Asadulghani, Nasirul Islam, K. M., and Mekalanos, J. J. (1999). Lysogenic conversion of environmental Vibrio mimicus strains by CTXPhi. Infect. Immun. 67, 5723-5729.

Feiner, R., Argov, T., Rabinovich, L., Sigal, N., Borovok, I., and Herskovits, A. A. (2015). A new perspective on lysogeny: prophages as active regulatory switches of bacteria. Nat. Rev. Microbiol. 13, 641-650. doi: 10.1038/nrmicro3527

Foulongne, V., Sauvage, V., Hebert, C., Dereure, O., Cheval, J., Gouilh, M. A., et al. (2012). Human skin microbiota: high diversity of DNA viruses identified on the human skin by high throughput sequencing. PLoS One 7:e38499. doi: 10.1371 /journal.pone. 0038499

Fung, T. C., Olson, C. A., and Hsiao, E. Y. (2017). Interactions between the microbiota, immune and nervous systems in health and disease. Nat. Neurosci. 20, 145-155. doi: 10.1038/nn.4476

Goldberg, G. W., Jiang, W., Bikard, D., and Marraffini, L. A. (2014). Conditional tolerance of temperate phages via transcription-dependent CRISPR-Cas targeting. Nature 514, 633-637. doi: 10.1038/nature 13637

Górski, A., Borysowski, J., Międzybrodzki, R., Weber-Dąbrowska, B., McGrath, S., and Van Sinderen, D. (2007). "Bacteriophages in medicine," in Bacteriophage: Genetics and Molecular Biology, eds S. McGrath, and D. van Sinderen (Norfolk: Caister Academic Press).

Górski, A., Dabrowska, K., Miedzybrodzki, R., Weber-Dabrowska, B., Lusiak-Szelachowska, M., Jonczyk-Matysiak, E., et al. (2017). Phages and immunomodulation. Future Microbiol. 12, 905-914. doi: 10.2217/fmb-20170049

Górski, A., Kniotek, M., Perkowska-Ptasinska, A., Mróz, A., Przerwa, A., Gorczyca, W., et al. (2006a). Bacteriophages and transplantation tolerance. Transpl. Proc. 38, 331-333. doi: 10.1016/j.transproceed.2005.12.073

Górski, A., Miedzybrodzki, R., Borysowski, J., Dabrowska, K., Wierzbicki, P., Ohams, M., et al. (2012). Phage as a modulator of immune responses: practical implications for phage therapy. Adv. Virus Res. 83, 41-71. doi: 10.1016/B978-012-394438-2.00002-5

Górski, A., Wazna, E., Dabrowska, B. W., Dabrowska, K., Switala-Jelen, K., and Miedzybrodzki, R. (2006b). Bacteriophage translocation. FEMS Immunol. Med. Microbiol. 46, 313-319. doi: 10.1111/j.1574-695X.2006.00044.x

Handley, S. A., Thackray, L. B., Zhao, G., Presti, R., Miller, A. D., Droit, L., et al. (2012). Pathogenic simian immunodeficiency virus infection is associated with expansion of the enteric virome. Cell 151, 253-266. doi: 10.1016/j.cell.2012. 09.024

Harrison, E., and Brockhurst, M. A. (2017). Ecological and evolutionary benefits of temperate phage: what does or doesn't kill you makes you stronger. Bioessays 39:1700112. doi: 10.1002/bies.201700112:

Hobbs, Z., and Abedon, S. T. (2016). Diversity of phage infection types and associated terminology: the problem with 'Lytic or lysogenic'. FEMS Microbiol. Lett. 363:fnw047. doi: 10.1093/femsle/fnw047

Holt, G. S., Lodge, J. K., McCarthy, A. J., Graham, A. K., Young, G., Bridge, S. H., et al. (2017). Shigatoxin encoding Bacteriophage varphi24B modulates bacterial metabolism to raise antimicrobial tolerance. Sci. Rep. 7:40424. doi: $10.1038 /$ srep 40424

Hooper, L. V., and Gordon, J. I. (2001). Commensal host-bacterial relationships in the gut. Science 292, 1115-1118. doi: 10.1126/science.1058709

Hsia, R., Ohayon, H., Gounon, P., Dautry-Varsat, A., and Bavoil, P. M. (2000). Phage infection of the obligate intracellular bacterium, Chlamydia psittaci strain guinea pig inclusion conjunctivitis. Microbes Infect. 2, 761-772. doi: 10.1016/ S1286-4579(00)90356-3

Huseyin, C. E., O’Toole, P. W., Cotter, P. D., and Scanlan, P. D. (2017). Forgotten fungi-the gut mycobiome in human health and disease. FEMS Microbiol. Rev. 41, 479-511. doi: 10.1093/femsre/fuw047

Ivanov, I. I., Frutos Rde, L., Manel, N., Yoshinaga, K., Rifkin, D. B., Sartor, R. B., et al. (2008). Specific microbiota direct the differentiation of IL-17-producing T-helper cells in the mucosa of the small intestine. Cell Host Microbe 4, 337-349. doi: 10.1016/j.chom.2008.09.009

Johnston, N. (2002). Viral Trojan horse for combating tuberculosis. Drug Discov. Today 7, 333-335. doi: 10.1016/S1359-6446(02)02222-5

Koskella, B., and Meaden, S. (2013). Understanding bacteriophage specificity in natural microbial communities. Viruses 5, 806-823. doi: 10.3390/v5030806
Koskinen, K., Pausan, M. R., Perras, A. K., Beck, M., Bang, C., Mora, M., et al. (2017). First insights into the diverse human archaeome: specific detection of archaea in the gastrointestinal tract, lung, and nose and on skin. mBio 8:e00824-17. doi: 10.1128/mBio.00824-17

Labrie, S. J., Samson, J. E., and Moineau, S. (2010). Bacteriophage resistance mechanisms. Nat. Rev. Microbiol. 8, 317-327. doi: 10.1038/nrmicro2315

Lehti, T. A., Pajunen, M. I., Skog, M. S., and Finne, J. (2017). Internalization of a polysialic acid-binding Escherichia coli bacteriophage into eukaryotic neuroblastoma cells. Nat. Commun. 8:1915. doi: 10.1038/s41467-01702057-3

Lekunberri, I., Subirats, J., Borrego, C. M., and Balcazar, J. L. (2017). Exploring the contribution of bacteriophages to antibiotic resistance. Environ. Pollut. $220(\mathrm{Pt}$ B), 981-984. doi: 10.1016/j.envpol.2016.11.059

Leskinen, K., Blasdel, B. G., Lavigne, R., and Skurnik, M. (2016). RNA-sequencing reveals the progression of phage-host interactions between phiR1-37 and Yersinia enterocolitica. Viruses 8:111. doi: 10.3390/v8040111

Majewska, J., Beta, W., Lecion, D., Hodyra-Stefaniak, K., Klopot, A., Kazmierczak, Z., et al. (2015). Oral application of T4 phage induces weak antibody production in the gut and in the blood. Viruses 7, 4783-4799. doi: 10.3390/v7082845

Manrique, P., Bolduc, B., Walk, S. T., van der Oost, J., de Vos, W. M., and Young, M. J. (2016). Healthy human gut phageome. Proc. Natl. Acad. Sci. U.S.A. 113, 10400-10405. doi: 10.1073/pnas.1601060113

Maslov, S., and Sneppen, K. (2017). Population cycles and species diversity in dynamic Kill-the-Winner model of microbial ecosystems. Sci. Rep. 7:39642. doi: $10.1038 /$ srep 39642

Matos, R. C., Lapaque, N., Rigottier-Gois, L., Debarbieux, L., Meylheuc, T., Gonzalez-Zorn, B., et al. (2013). Enterococcus faecalis prophage dynamics and contributions to pathogenic traits. PLoS Genet. 9:e1003539. doi: 10.1371/ journal.pgen.1003539

Mazmanian, S. K., Liu, C. H., Tzianabos, A. O., and Kasper, D. L. (2005). An immunomodulatory molecule of symbiotic bacteria directs maturation of the host immune system. Cell 122, 107-118. doi: 10.1016/j.cell.2005.05.007

McVay, C. S., Velasquez, M., and Fralick, J. A. (2007). Phage therapy of Pseudomonas aeruginosa infection in a mouse burn wound model. Antimicrob. Agents Chemother. 51, 1934-1938. doi: 10.1128/AAC.01028-06

Metcalf, J. A., Funkhouser-Jones, L. J., Brileya, K., Reysenbach, A. L., and Bordenstein, S. R. (2014). Antibacterial gene transfer across the tree of life. eLife 3:e04266. doi: 10.7554/eLife.04266

Miller-Ensminger, T., Garretto, A., Brenner, J., Thomas-White, K., Zambom, A., Wolfe, A. J., et al. (2018). Bacteriophages of the urinary microbiome. J. Bacteriol. 200:e00738-17. doi: 10.1128/JB.00738-17

Minot, S., Sinha, R., Chen, J., Li, H., Keilbaugh, S. A., Wu, G. D., et al. (2011). The human gut virome: inter-individual variation and dynamic response to diet. Genome Res. 21, 1616-1625. doi: 10.1101/gr.122705.111

Mojardin, L., and Salas, M. (2016). Global transcriptional analysis of virushost interactions between phage varphi29 and Bacillus subtilis. J. Virol. 90, 9293-9304. doi: 10.1128/JVI.01245-16

Moran, Y., Fredman, D., Szczesny, P., Grynberg, M., and Technau, U. (2012). Recurrent horizontal transfer of bacterial toxin genes to eukaryotes. Mol. Biol. Evol. 29, 2223-2230. doi: 10.1093/molbev/mss089

Nanda, A. M., Thormann, K., and Frunzke, J. (2015). Impact of spontaneous prophage induction on the fitness of bacterial populations and host-microbe interactions. J. Bacteriol. 197, 410-419. doi: 10.1128/JB.02230-14

Nedialkova, L. P., Sidstedt, M., Koeppel, M. B., Spriewald, S., Ring, D., Gerlach, R. G., et al. (2016). Temperate phages promote colicin-dependent fitness of Salmonella enterica serovar Typhimurium. Environ. Microbiol. 18, 1591-1603. doi: 10.1111/1462-2920.13077

Nguyen, S., Baker, K., Padman, B. S., Patwa, R., Dunstan, R. A., Weston, T. A., et al. (2017). Bacteriophage transcytosis provides a mechanism to cross epithelial cell layers. mBio 8:e01874-17. doi: 10.1128/mBio.01874-17

Norman, J. M., Handley, S. A., Baldridge, M. T., Droit, L., Liu, C. Y., Keller, B. C., et al. (2015). Disease-specific alterations in the enteric virome in inflammatory bowel disease. Cell 160, 447-460. doi: 10.1016/j.cell.2015.01.002

Novince, C. M., Whittow, C. R., Aartun, J. D., Hathaway, J. D., Poulides, N., Chavez, M. B., et al. (2017). Commensal gut microbiota immunomodulatory actions in bone marrow and liver have catabolic effects on skeletal homeostasis in health. Sci. Rep. 7:5747. doi: 10.1038/s41598-017-06126-x 
Obeng, N., Pratama, A. A., and Elsas, J. D. (2016). The significance of mutualistic phages for bacterial ecology and evolution. Trends Microbiol. 24, 440-449. doi: 10.1016/j.tim.2015.12.009

Oh, J., Byrd, A. L., Deming, C., Conlan, S., Program, N. C. S., Kong, H. H., et al. (2014). Biogeography and individuality shape function in the human skin metagenome. Nature 514, 59-64. doi: 10.1038/nature13786

Oh, J., Byrd, A. L., Park, M., Program, N. C. S., Kong, H. H., and Segre, J. A. (2016). Temporal stability of the human skin microbiome. Cell 165, 854-866. doi: 10.1016/j.cell.2016.04.008

Palmer, K. L., and Gilmore, M. S. (2010). Multidrug-resistant enterococci lack CRISPR-cas. mBio 1:e00227-10. doi: 10.1128/mBio.00227-10

Pleska, M., Lang, M., Refardt, D., Levin, B. R., and Guet, C. C. (2018). Phage-host population dynamics promotes prophage acquisition in bacteria with innate immunity. Nat. Ecol. Evol. 2, 359-366. doi: 10.1038/s41559-017-0424-z

Pride, D. T., Salzman, J., Haynes, M., Rohwer, F., Davis-Long, C., White, R. A., et al. (2012). Evidence of a robust resident bacteriophage population revealed through analysis of the human salivary virome. ISME J. 6, 915-926. doi: 10.1038/ismej.2011.169

Rabinovich, L., Sigal, N., Borovok, I., Nir-Paz, R., and Herskovits, A. A. (2012). Prophage excision activates Listeria competence genes that promote phagosomal escape and virulence. Cell 150, 792-802. doi: 10.1016/j.cell.2012. 06.036

Raymann, K., Moeller, A. H., Goodman, A. L., and Ochman, H. (2017). Unexplored archaeal diversity in the great ape gut microbiome. mSphere 2:e00026-17. doi: $10.1128 / \mathrm{mSphere}$.00026-17

Reyes, A., Haynes, M., Hanson, N., Angly, F. E., Heath, A. C., Rohwer, F., et al. (2010). Viruses in the faecal microbiota of monozygotic twins and their mothers. Nature 466, 334-U381. doi: 10.1038/nature09199

Rice, S. A., Tan, C. H., Mikkelsen, P. J., Kung, V., Woo, J., Tay, M., et al. (2009). The biofilm life cycle and virulence of Pseudomonas aeruginosa are dependent on a filamentous prophage. ISME J. 3, 271-282. doi: 10.1038/ismej.2008.109

Roach, D. R., Leung, C. Y., Henry, M., Morello, E., Singh, D., Di Santo, J. P., et al. (2017). Synergy between the host immune system and bacteriophage is essential for successful phage therapy against an acute respiratory pathogen. Cell Host Microbe 22, 38.e3-47.e3. doi: 10.1016/j.chom.2017.06.018

Rodriguez-Brito, B., Li, L. L., Wegley, L., Furlan, M., Angly, F., Breitbart, M., et al. (2010). Viral and microbial community dynamics in four aquatic environments. ISME J. 4, 739-751. doi: 10.1038/ismej.2010.1

Roossinck, M. J. (2011). The good viruses: viral mutualistic symbioses. Nat. Rev. Microbiol. 9, 99-108. doi: 10.1038/nrmicro2491

Rosenwald, A. G., Murray, B., Toth, T., Madupu, R., Kyrillos, A., and Arora, G. (2014). Evidence for horizontal gene transfer between Chlamydophila pneumoniae and Chlamydia phage. Bacteriophage 4:e965076. doi: 10.4161/ 21597073.2014.965076

Royle, L., Matthews, E., Corfield, A., Berry, M., Rudd, P. M., Dwek, R. A., et al. (2008). Glycan structures of ocular surface mucins in man, rabbit and dog display species differences. Glycoconj. J. 25, 763-773. doi: 10.1007/s10719-0089136-6

Santiago-Rodriguez, T. M., Ly, M., Bonilla, N., and Pride, D. T. (2015). The human urine virome in association with urinary tract infections. Front. Microbiol. 6:14. doi: $10.3389 /$ fmicb. 2015.00014

Schmidt, H., Bielaszewska, M., and Karch, H. (1999). Transduction of enteric Escherichia coli isolates with a derivative of Shiga toxin 2-encoding bacteriophage phi3538 isolated from Escherichia coli O157:H7. Appl. Environ. Microbiol. 65, 3855-3861.

Schnupf, P., Gaboriau-Routhiau, V., Sansonetti, P. J., and Cerf-Bensussan, N. (2017). Segmented filamentous bacteria, Th17 inducers and helpers in a hostile world. Curr. Opin. Microbiol. 35, 100-109. doi: 10.1016/j.mib.2017. 03.004

Secor, P. R., Michaels, L. A., Smigiel, K. S., Rohani, M. G., Jennings, L. K., Hisert, K. B., et al. (2017). Filamentous bacteriophage produced by Pseudomonas aeruginosa alters the inflammatory response and promotes noninvasive infection in vivo. Infect. Immun. 85:e00648-16. doi: 10.1128/IAI.00648-16

Secor, P. R., Sweere, J. M., Michaels, L. A., Malkovskiy, A. V., Lazzareschi, D., Katznelson, E., et al. (2015). Filamentous bacteriophage promote biofilm assembly and function. Cell Host Microbe 18, 549-559. doi: 10.1016/j.chom. 2015.10 .013
Shaikh, N., and Tarr, P. I. (2003). Escherichia coli O157:H7 Shiga toxinencoding bacteriophages: integrations, excisions, truncations, and evolutionary implications. J. Bacteriol. 185, 3596-3605. doi: 10.1128/JB.185.12.3596-3605. 2003

Sorek, R., Kunin, V., and Hugenholtz, P. (2008). CRISPR-a widespread system that provides acquired resistance against phages in bacteria and archaea. Nat. Rev. Microbiol. 6, 181-186. doi: 10.1038/nrmicro1793

Thingstad, T. F. (2000). Elements of a theory for the mechanisms controlling abundance, diversity, and biogeochemical role of lytic bacterial viruses in aquatic systems. Limnol. Oceanogr. 45, 1320-1328. doi: 10.4319/lo.2000.45.6. 1320

Tian, Y., Wu, M., Liu, X., Liu, Z., Zhou, Q., Niu, Z., et al. (2015). Probing the endocytic pathways of the filamentous bacteriophage in live cells using ratiometric $\mathrm{pH}$ fluorescent indicator. Adv. Healthc. Mater. 4, 413-419. doi: $10.1002 /$ adhm. 201400508

Tock, M. R., and Dryden, D. T. (2005). The biology of restriction and antirestriction. Curr. Opin. Microbiol. 8, 466-472. doi: 10.1016/j.mib.2005.06.003

Touchon, M., Moura de Sousa, J. A., and Rocha, E. P. (2017). Embracing the enemy: the diversification of microbial gene repertoires by phage-mediated horizontal gene transfer. Curr. Opin. Microbiol. 38, 66-73. doi: 10.1016/j.mib.2017.04.010

van Belkum, A., Soriaga, L. B., LaFave, M. C., Akella, S., Veyrieras, J. B., Barbu, E. M., et al. (2015). Phylogenetic distribution of CRISPR-Cas Systems in antibiotic-resistant Pseudomonas aeruginosa. mBio 6:e01796-15. doi: 10.1128/ mBio.01796-15

Virgin, H. W. (2014). The virome in mammalian physiology and disease. Cell 157, 142-150. doi: 10.1016/j.cell.2014.02.032

Wagner, J., Maksimovic, J., Farries, G., Sim, W. H., Bishop, R. F., Cameron, D. J., et al. (2013). Bacteriophages in gut samples from pediatric Crohn's disease patients: metagenomic analysis using 454 pyrosequencing. Inflamm. Bowel Dis. 19, 1598-1608. doi: 10.1097/MIB.0b013e318292477c

Wahida, A., Ritter, K., and Horz, H. P. (2016). The janus-face of bacteriophages across human body habitats. PLoS Pathog. 12:e1005634. doi: 10.1371/journal. ppat.1005634

Weinbauer, M. G. (2004). Ecology of prokaryotic viruses. FEMS Microbiol. Rev. 28, 127-181. doi: 10.1016/j.femsre.2003.08.001

Willner, D., Furlan, M., Haynes, M., Schmieder, R., Angly, F. E., Silva, J., et al. (2009). Metagenomic analysis of respiratory tract DNA viral communities in cystic fibrosis and non-cystic fibrosis individuals. PLoS One 4:e7370. doi: 10.1371/journal.pone.0007370

Willner, D., Furlan, M., Schmieder, R., Grasis, J. A., Pride, D. T., Relman, D. A., et al. (2011). Metagenomic detection of phage-encoded platelet-binding factors in the human oral cavity. Proc. Natl. Acad. Sci. U.S.A. 108(Suppl. 1), 4547-4553. doi: 10.1073/pnas.1000089107

Zhang, L., Sun, L., Wei, R., Gao, Q., He, T., Xu, C., et al. (2017). Intracellular Staphylococcus aureus control by virulent bacteriophages within MAC-T bovine mammary epithelial cells. Antimicrob. Agents Chemother. 61, doi: 10.1128/AAC. 01990-16

Zhao, G., Vatanen, T., Droit, L., Park, A., Kostic, A. D., Poon, T. W., et al. (2017). Intestinal virome changes precede autoimmunity in type I diabetes-susceptible children. Proc. Natl. Acad. Sci. U.S.A. 114, E6166-E6175. doi: 10.1073/pnas. 1706359114

Zhao, Q., and Elson, C. O. (2018). Adaptive immune education by gut microbiota antigens. Immunology 54, 28-37. doi: 10.1111/imm.12896

Zou, S., Caler, L., Colombini-Hatch, S., Glynn, S., and Srinivas, P. (2016). Research on the human virome: where are we and what is next. Microbiome 4:32. doi: 10.1186/s40168-016-0177-y

Conflict of Interest Statement: The authors declare that the research was conducted in the absence of any commercial or financial relationships that could be construed as a potential conflict of interest.

Copyright (c) 2018 Chatterjee and Duerkop. This is an open-access article distributed under the terms of the Creative Commons Attribution License (CC BY). The use, distribution or reproduction in other forums is permitted, provided the original author(s) and the copyright owner are credited and that the original publication in this journal is cited, in accordance with accepted academic practice. No use, distribution or reproduction is permitted which does not comply with these terms. 